\title{
Una mirada sobre el impacto del uso de las redes sociales en las empresas argentinas
}

\author{
Guillermo Alberto Tricoci, Ariana Rosenthal, \\ Pablo Corral y Pablo Gil \\ Universidad de Buenos Aires, Argentina
}

Recibido: 19 de marzo de 2013

Aceptado: 5 de noviembre de 2013

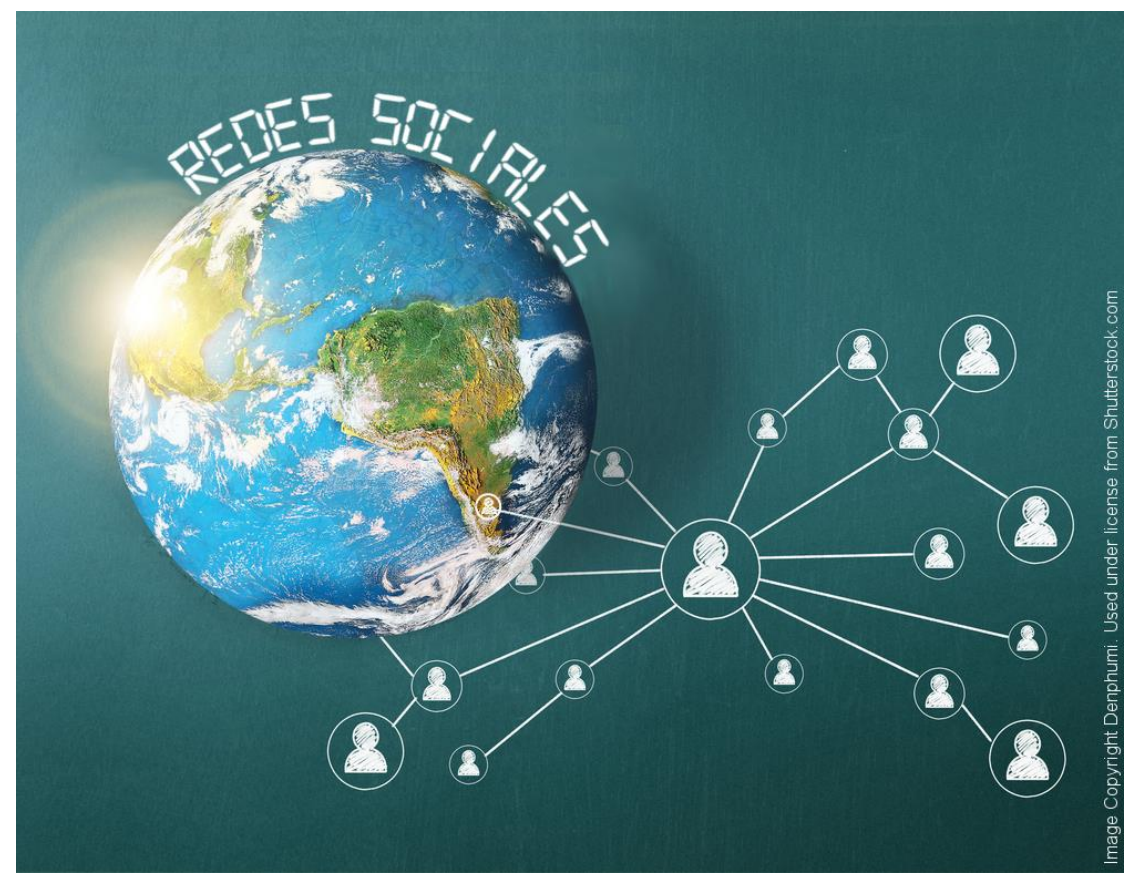

\begin{abstract}
Social networks are changing the way people relate to each other. Understating the impact of use of social network in business and the adoption process of these new technologies in organizations is the focus of this article. We present the results of our investigation and research during 2012 on the use of social networks in enterprises of Argentina, the assessment of its impact of use on the sales of these companies, and in improving their competitiveness. We are also comparing the degree of development of social networks with other e-commerce applications and with the use of internet. Finally, we raise the need to investigate the negative impact that may arise from the use of these technologies, such us adverse or discredit campaigns.
\end{abstract}

KEY WORDS: Social Networks, Competitiveness, ICT

\section{RESUMEN}

Las redes sociales están cambiando la forma en que se relacionan socialmente los individuos. El foco del presente trabajo es entender el impacto del uso de las redes sociales en los negocios y cómo se dan los procesos de adopción de estas nuevas tecnologías en las organizaciones. Presentamos los resultados de la investigación que hemos desarrollado durante el año 2012 sobre el uso de las redes sociales en las empresas argentinas, la valoración de su impacto de uso en las ventas de dichas compañías, y en la mejora de su competitividad. Realizamos además una comparamos del grado de desarrollo de las redes sociales respecto de otras aplicaciones de comercio electrónico y del uso de los sitios de internet. Finalmente, dejamos planteadas la necesidad de estudiar los efectos negativos que se pueden presentar por el uso de estas tecnologías, como por ejemplo el inicio de campañas adversas o de desprestigio.

PALABRAS CLAVES: Redes sociales, competitividad, TIC 


\section{Introducción}

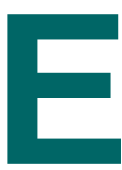

1 desarrollo de las redes sociales está cambiando la forma como se relacionan los individuos, produciendo impacto tanto en la vida cotidiana de los mismos como en la de la sociedad en general. Actualmente, utilizamos las redes sociales para saludarnos por cumpleaños, por acontecimientos importantes, compartir detalles de viajes, eventos, buscar trabajo, darnos a conocer, contar qué estamos haciendo, etc. Rifkin (2011) describe con suma claridad cómo los motores de búsqueda lateral y los sitios de redes sociales han cambiado incluso nuestro modo de trabajar, existiendo hoy un nuevo espacio distribuido y colaborativo para compartir conocimiento y para estimular la creatividad y la innovación en todos los terrenos.

Este fuerte cambio en nuestras vidas está llegando también a las empresas. Pero, ¿cuál es el impacto del uso de las redes sociales en los negocios, y cómo se dan los procesos de adopción de estas nuevas tecnologías en las organizaciones?

Vivimos en un mundo donde el sector de TIC (Tecnologías de la Información y las Comunicaciones) se muestra bastante dinámico, con diferencias importantes entre regiones. Se estima un producto del orden de los 2.6 billones de euros según el Observatorio de Tecnología de la Información Europeo (EITO) (2008), con un crecimiento importante en el área de los dispositivos móviles.

Indicadores del Foro Económico Mundial muestran un mayor grado de uso de las TIC en los individuos que en las empresas y los gobiernos, lo que indicaría que la adopción de nuevas tecnologías es más rápida a nivel individual que organizacional. De acuerdo con el Índice NRI (Networked Readiness Index) en el Reporte Global de Tecnología de la Información 2012, para Argentina, el grado de uso de las TIC en los individuos es 3.59, mientras que en las empresas llega a 3.42 y en el gobierno, a 3.12. Desde el punto de vista del ranking mundial del
NRI, estos índices determinan que la Argentina se encuentra en la posición 58 sobre 142 países en grado de uso de individuos, en el puesto 86 sobre 142 países en grado de uso de las empresas y en el lugar 119 sobre 142 países en grado de uso del gobierno ${ }^{1}$.

Desde el punto de vista organizacional, si bien los beneficios que producen las TIC en mejoras de costos están claramente identificados, la relación es mucho más difícil de establecer cuando se intenta extender a la diferenciación de productos y servicios que ofrecen las organizaciones.

En Tricoci (2011), se analizan distintos estados evolutivos de las organizaciones con respecto al grado de complejidad de las TICs implementadas, planteándose la existencia de distintas etapas formadas por factores internos y externos. De esta manera, se pueden distinguir estadios de "Registro", "Análisis de Información" y "Trabajo Cooperativo". Podríamos decir que el uso intensivo de Internet y de las redes sociales se asocia al estadio de "Trabajo Cooperativo" y, por ende, su adopción requeriría empresas en un estado evolutivo de esa característica.

En Yoguel et al (2004) se relaciona la difusión de las TIC y las competencias endógenas de las firmas en la Argentina. El trabajo indica que sólo el $17 \%$ de las firmas poseían alta difusión y competencias endógenas, el $21 \%$ mostraba baja difusión y competencias endógenas y el resto, disparidades entre ambos elementos. No necesariamente un alto grado de inversión en TIC se encuentra directamente relacionado con un aprovechamiento de sus potencialidades productivas y diferenciales.

Existen estudios previos a nivel oficial sobre temas de innovación y uso e implementación de las TIC, entre los que podemos mencionar:

\footnotetext{
${ }^{1}$ Reporte Global de tecnología de la Información 2012 , página 15 , tabla 4 sub índice de uso y sus pilares. Cabe aclarar que las valoraciones son entre 7 y 1 .
} 
- La Encuesta Nacional de Innovación y Conducta Tecnológica de las empresas argentinas, publicada en 2003 y coordinada por el Instituto Nacional de Estadísticas y Censos (INDEC) de la República Argentina. Recopiló datos del período 1998-2001 en lo referente a las actividades generales de innovación.

- La Encuesta Nacional a Empresas sobre Innovación, I+D y TIC, publicada en 2006 y coordinada por la Secretaría de Ciencia, Tecnología e Innovación Productiva de la República Argentina. Incluye el período 20022004 en lo referente a las actividades generales de innovación.

- La Encuesta Nacional sobre Innovación y Conducta Tecnológica (ENIT) realizada durante el año 2005 por el Instituto Nacional de Estadísticas y Censos (INDEC) de la República Argentina, y publicada el 11 de septiembre de 2006.

- La Encuesta Nacional sobre Innovación y Conducta Tecnológica (ENIT) realizada durante el año 2006 por el Instituto Nacional de Estadísticas y Censos (INDEC) de la República Argentina, y publicada el 11 de julio de 2007.

- La Encuesta Nacional sobre Innovación y Conducta Tecnológica (ENIT) realizada durante el año 2007 por el Instituto Nacional de Estadísticas y Censos (INDEC) de la República Argentina, y publicada el 29 de diciembre de 2008.

Estas fuentes de datos tienen en cuenta el uso de las aplicaciones WEB y el comercio electrónico y nos permiten realizar consideraciones sobre su evolución ${ }^{2}$.

Mientras escribíamos el presente trabajo, se publicó un artículo periodístico titulado "La venganza de Pareto en las redes sociales", en un diario dominical en Buenos Aires. Este artículo presenta la conocida teoría del 80 / 20 de Wilfredo Pareto que ha sido usada en múltiples

\footnotetext{
${ }^{2}$ Estos antecedentes no aplican al tema de las redes sociales y su impacto.
}

campos y situaciones., Anderson (2004) ${ }^{3}$ la aplicó para relacionar los sitios de Internet y su frecuencia de acceso. Esta relación mostraba que se puede ver una gran cantidad de sitios con muy baja frecuencia de acceso y unos pocos sitios con relevantes frecuencias de acceso. Recientes trabajos sobre el uso de Twitter y Facebook muestran que, en el primero, el $2 \%$ de los usuarios maneja el $70 \%$ de los mensajes y que, en el segundo, el $20 \%$ de usuarios triplican en uso al $80 \%$ restante.

Somos un equipo de docentes universitarios que nos encontramos desarrollando un proyecto de investigación bajo el título "Impacto de las TIC en la competitividad", en la Facultad de Ciencias Económicas de la Universidad de Buenos Aires (República Argentina). Durante su desarrollo, y como parte de la metodología de recolección de datos, implementamos una encuesta en la cual incluimos un grupo de preguntas referidas a las redes sociales e Internet, para conocer datos de uso y valoraciones de los impactos de dichas tecnologías en las ventas de las firmas encuestadas. Varias de estas preguntas se relacionan más con objetivos secundarios del proyecto y con el fin de obtener datos para formular hipótesis en futuros proyectos.

En nuestro trabajo, al analizar los datos obtenidos, nos encontramos con algunas "sorpresas", que describimos en detalle en este artículo.

\section{Desarrollo del trabajo realizado}

Los resultados que exponemos surgen de una encuesta propia del proyecto, que contiene 7 grupos de preguntas como lo indica la Tabla 1.

\footnotetext{
${ }^{3}$ Anderson Chris (2004) popularizó en la revista Wired y luego con su libro y sitio web "The long Tail", el concepto de la cola larga.
} 
Tabla 1. El conjunto de preguntas específicas a las cuales se refiere el trabajo se encuentran en el grupo "Uso de las redes sociales y la web"

\begin{tabular}{|c|l|}
\hline Rubro & \\
\hline 1 & Identificación de la Firma \\
\hline 2 & Identificación de la Función de Sistema \\
\hline 3 & $\begin{array}{l}\text { Proceso de la toma de decisiones de } \\
\text { TICs }\end{array}$ \\
\hline 4 & Uso de las redes sociales y la web \\
\hline 5 & Inversiones en TICs \\
\hline 6 & $\begin{array}{l}\text { Actividades de investigación y } \\
\text { desarrollo }\end{array}$ \\
\hline 7 & Uso de las TIC \\
\hline
\end{tabular}

\section{Referidas a las Redes Sociales}

¿Su firma tiene presencia en las redes sociales? $¿$ Tiene presencia en Facebook, YouTube, y/ o Twitter?

¿Tiene presencia en otras no mencionadas?

Valore de 7 a 1 (siendo 7 excelente y 1 nulo) el impacto que tiene en sus ventas la presencia en las redes sociales.

¿Ha medido el impacto que producen en sus ventas la presencia en las redes sociales?

\section{Referidas a otras aplicaciones web:}

1. ¿Su firma tiene sitio en Internet?

2. ¿Realiza compras por medios automáticos, entendiéndose estos por cualquier medio electrónico desde el correo electrónico hasta otros de B2B?.

3. ¿Realiza sus ventas medios automáticos? Cuáles?

La encuesta fue realizada en más de 100 empresas de la Ciudad Autónoma de Buenos Aires y Gran Buenos Aires (Región metropolitana y más densamente poblada de la República Argentina) durante el año 2012.

\section{Resultados obtenidos}

Nuestro grupo realizó una primera aproximación a la interpretación de estos resultados en el VI Congreso Internacional de Economía y Gestión 2012, llevado a cabo en la Facultad de Ciencias Económicas de la Universidad de Buenos Aires (Argentina) en octubre de ese año.

Las firmas que tienen presencia en las redes sociales son algo menos del $50 \%$ de la muestra.

El 78\% de firmas que están presentes en las redes sociales pertenecen al sector de servicios y el $22 \%$ restantes son manufactureras. La red social más usada es Facebook, con casi el $90 \%$ de adeptos. La Figura 1 muestra la distribución de las respuestas entre las distintas redes sociales y su combinación de uso ${ }^{4}$.

\section{Figura 1}

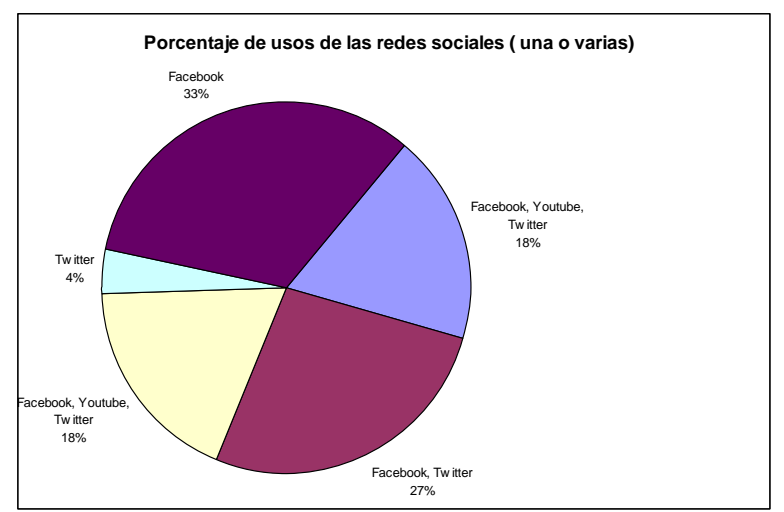

Otra de las preguntas sugería que mencionaran otras redes sociales que utilizaban, pero no incluidas en la lista anterior: $\mathrm{La}$ red profesional LinkedIn fue la más elegida con el 12 $\%$. ¿Cuáles fueron las valoraciones de impacto de las redes sociales sobre las ventas (siendo la valoración 7 excelente y 1 nulo)?. Se resumen las valoraciones en la Tabla 2

\footnotetext{
4 Los encuestados estaban habilitados a elegir más de una red. Es usual ver que las empresas estén en más de una red social.
} 
Tabla 2: Valores promedio y desvíos sobre impacto de redes sociales en las ventas de firmas participantes en redes sociales.

\begin{tabular}{|l|c|c|}
\hline \multicolumn{1}{|c|}{ Agrupación } & $\begin{array}{c}\text { Valor } \\
\text { promedio }\end{array}$ & $\begin{array}{c}\text { Desvío } \\
\text { estándar }\end{array}$ \\
\hline $\begin{array}{l}\text { Firmas que están en } \\
\text { la red }\end{array}$ & 2.8 & 1.6 \\
\hline $\begin{array}{l}\text { Firmas del sector de } \\
\text { manufactura de la } \\
\text { muestra }\end{array}$ & 3.2 & 2.8 \\
\hline
\end{tabular}

El valor promedio de la valoración es de 2,80 , con un desvío estándar de $1,6^{5}$. Si separamos las firmas de manufactura, encontramos que el valor promedio es algo mayor (3.20), pero el desvío también es más alto y se ubica en 2.80. Esto está en el orden de magnitud del total de la muestra, indicando que la percepción de impacto en las ventas parecería no ser importante. No se logra llegar a un media de 3.5, valor medio de las valoraciones entre $1 \mathrm{y}$ 7.

Avanzando un poco más, intentamos llevar la percepción o valoración de impacto en las ventas a la medición cuantitativa, mediante la realización y análisis de la siguiente pregunta: ¿midieron el impacto del uso de las redes sociales sobre las ventas? Veamos el resultado obtenido en la Figura 2.

Si armamos un subgrupo de firmas que están en las redes sociales e hicieron una medición del impacto de las mismas sobre las ventas, ¿cómo respondieron estas a las valoraciones? La valoración promedio de este subgrupo sobre su percepción del impacto sobre las ventas es algo más alto (3.81). Obsérvese que si bien la valoración promedio es más alta, tan solo un punto más, excede ligeramente al punto medio $(3,5)$.
Figura 2

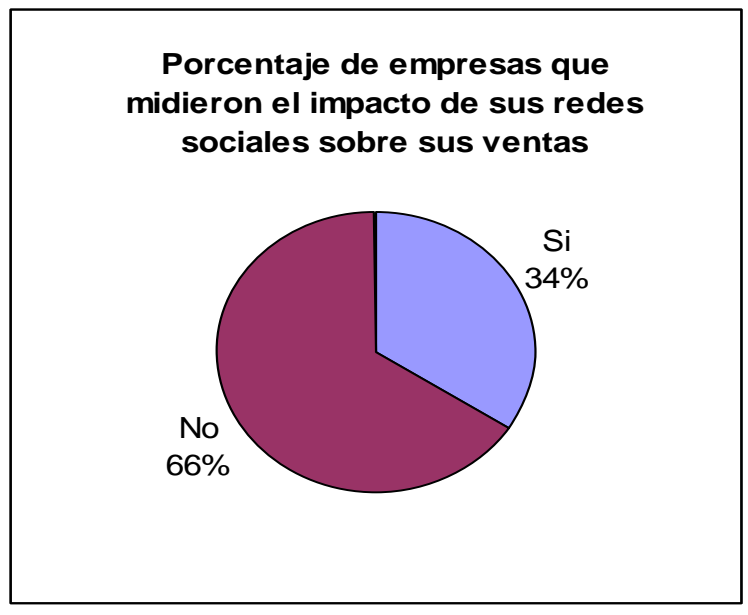

Los que midieron cuantitativamente el impacto, si bien presentan una valoración mayor, no logran mostrar un resultado significativamente más elevado.

Cuando se realizan las combinaciones por tamaño de empresa o composición del capital, no se aprecian cambios significativos en los resultados.

Ahora, veamos qué le sucede a las firmas de la muestra con otras tecnologías disponibles, como la existencia de sus propios sitios de internet, la realización de compras por medios electrónicos y las ventas por dichos medios. Se presentan los resultados en la Tabla 3.

Tabla 3. Resultados del uso de otras aplicaciones de TIC.

\begin{tabular}{|l|c|c|}
\hline & $\begin{array}{c}\text { Total de } \\
\text { la } \\
\text { muestra }\end{array}$ & $\begin{array}{c}\text { Las que } \\
\text { respondieron } \\
\text { que estaban en } \\
\text { las redes sociales }\end{array}$ \\
\hline ¿Tiene sitio de Internet? & $92 \%$ & $100 \%$ \\
\hline $\begin{array}{l}\text { iRealiza sus compras por } \\
\text { algún medio electrónico } \\
\text { (desde B2B hasta un e- } \\
\text { mail)? }\end{array}$ & $82 \%$ & $92 \%$ \\
\hline $\begin{array}{l}\text { ¿Realiza sus ventas } \\
\text { (totales o parciales) por } \\
\text { medio electrónico? }\end{array}$ & $52 \%$ & $52 \%$ \\
\hline
\end{tabular}

\footnotetext{
${ }^{5}$ La moda y la mediana fue 3 .
} 
Estos resultados muestran que las firmas incluidas en la muestra utilizan otras aplicaciones web en un grado mucho mayor que su presencia en las redes sociales.

La disponibilidad de sitios web propios es total en el grupo que están en las redes sociales $(100 \%$,$) y en el caso del total de la muestra, es$ superior al $90 \%$.

El uso de algún medio electrónico para realizar compras es muy alto, siendo superior en las que tiene presencia en las redes.

Las ventas electrónicas tienen un grado de utilización menor y sin diferencias entre las que están en las redes sociales y el total de la muestra. Cabe acotar que si analizáramos una serie histórica podríamos ver que el aumento en el tiempo del uso de medios electrónicos para las ventas muestra un crecimiento mucho menor al referido a su uso en las compras.

Finalmente se preguntó a las firmas encuestadas sobre su valoración respecto de las inversiones en las TIC en el aumento de competitividad en su firma y en la economía argentina en general. Si se comparan los resultados de estas dos valoraciones en la muestra total y el subgrupo de los que están en las redes, se puede apreciar que el subgrupo que participa activamente en las redes sociales tiene sólo una valoración superior en un $5 \%$ al total. Esto muestra que no es percibido como un impacto decisivo en la mejora de la competitividad.

\section{Conclusiones}

La amplia difusión de las redes sociales en el plano de los individuos, que está caracterizando a un grupo amplio de la población mayoritariamente joven, que accede y se comunica por estas redes, opera como un impulso para las empresas que recorren similares caminos. Hoy, el concepto de movilidad potencia esta difusión ya que las personas están permanentemente "conectadas", generalmente a través de dispositivos móviles con gran capacidad de procesamiento, y desean estar actualizados, leer y generar mensajes y contenido mientras se desplazan, navegan y se sienten, "libres". En el caso de las firmas el proceso se realiza a una velocidad distinta.

La decisión de tener presencia en las redes sociales, si bien es una decisión estratégica de una firma, no requiere grandes inversiones adicionales, dado que las plataformas tecnológicas básicas ya están disponibles.

El hecho de encontrar mayoritariamente empresas de servicios en las firmas que usan las redes sociales puede referirse a que los servicios tienen más altos componentes de bienes de información y de comunicación que las firmas de manufactura. La potencialidad de diversificar por el conocimiento, cercanía y gustos de los clientes es más fuerte y de mayor variabilidad en los servicios. Pero es contradictorio el resultado que las manufactureras tengan una valoración mayor que el total de la muestra, lo cual deja a las firmas de servicios con una valoración inferior a la total. De cualquier forma la valoración baja (por debajo del promedio de 3.5), podría indicar que estar en las redes tiene que ver más con una cuestión de imagen, características de sus clientes, intangibles o que simplemente las firmas vean a las redes sociales como un lugar en el que se deba estar, aún sin una clara visión o indicios de obtener diferencias competitivas sustentables, por lo menos hasta ahora. Por otro lado, como las restricciones y barreras de entrada son bajas y no se requieren inversiones muy relevantes, si están en una estadio de conectividad razonable, la gran mayoría podría estar presente y por lo tanto no sería una diferencia para ninguna. Pero por otro lado, clientes/usuarios de nivel TIC medio / alto ${ }^{6}$, pueden percibir la falta de presencia de un proveedor en las redes sociales como una limitación fuerte.

Dentro de las firmas que midieron el impacto de las redes sociales en las ventas, la valoración es un poco más alta en promedio, casi 4, pero aparece una valoración de mayor impacto en las ventas. El uso de las redes

\footnotetext{
${ }^{6}$ Son usuarios que están conectados, que usan dispositivos móviles, que prefieren las transacciones electrónicas a las manuales, etc.
} 
sociales en las organizaciones que componen la muestra presenta la idea que la tecnología está disponible, difundida en la mitad de los casos, pero que sus impactos se están "descubriendo". En los procesos de adopción de nueva tecnología, las firmas pasan por períodos de aprendizaje, hasta llegar a un mejor uso de la misma, ya que transitan un proceso de "Learning by doing" ("Aprender haciendo") para llegar a mejores niveles de aprovechamiento.

Es relativamente sencillo tener presencia en las redes sociales, pero parece que se están aún desarrollando nuevas potencialidades para sacar un resultado económico de las mismas.

Cuando miramos el uso de otras aplicaciones de las TIC, como la disponibilidad de sitios web, y las compras y ventas electrónicas, encontramos un grado de uso sustancialmente mayor. Podemos ver que dichas aplicaciones se encuentran en un estado evolutivo mayor que el uso de las redes sociales, las cuales no parece ser percibidas aún como una herramienta fundamental en la mejora en la competitividad

Adicionalmente, si bien no son aspectos directamente relacionados con las hipótesis y objetivos que hemos planteado como equipo de investigación, entendemos que ciertas organizaciones podrían retrasar su presencia en las redes sociales o estudiar detalladamente su mejor forma de adopción debido a potenciales impactos negativos de las mismas. Las redes sociales permiten a los clientes participar en línea y activamente de "conversaciones digitales", y tal como demuestran su satisfacción por los productos o servicios ofrecidos por las organizaciones, podrían ser el punto inicial de campañas publicitarias adversas en caso de insatisfacción. Otro riesgo a considerar está dado por la facilidad con la que competidores desleales conseguirían llevar a cabo acciones de desprestigio $^{7}$.

\footnotetext{
${ }^{7}$ Estas posibles externalidades negativas serán incluidas en próximos trabajos de nuestro grupo de investigación.
}

\section{Referencias}

Anderson C. "The long Tail" sitio web http://www.thelongtail.com/the_long_tail.

Guyot, C. (2013, Febrero 24). La venganza de Pareto en las redes sociales. Diario La Nación.

Rifkin, J. (2011). La tercera revolución industrial. Cómo el poder lateral está transformando la energía, la economía y el mundo. Paidós.

Tricoci, G. (2011). Las TIC y el conocimiento. Un enfoque económico y de negocios. (2da edición). Ediciones Cooperativas.

Tricoci, G., Rosenthal, A., Corral, P. \& Gil, P. (2012, Octubre). Impacto de las Tic en la Competitividad de las Firmas. Trabajo presentado y expuesto en el VI Congreso Internacional de Economía y Gestión: Econ 2012 en la Facultad de Ciencias Económicas de la Universidad de Buenos Aires, Buenos Aires, Argentina.

Yoguel, G., Novick, M., Milesi, D., Roitter, S. \& Borillo, J. (2004). Información y conocimiento: la difusión de TICs en la industria manufacturera argentina. Revista de CEPAL, Nro 82 página 139 a 152 .

World Economic Forum (2012). Reporte Global de Tecnología de la Información 2012 - The Global Information Technology Report 2012: Living in a Hyperconnected World. INSEAD.

\section{Información del autor de correspondencia}

Guillermo A. Tricoci.

tricoci@economicas.uba.ar.

Contador Público. Licenciado en Administración de Empresas Postgrado en Economía. Sub-Director del Departamento Pedagógico de Sistemas, Facultad de Ciencias Económicas, Universidad de Buenos Aires. Profesor Titular (int) de Tecnología de la Información. Director de Proyectos de Investigación en TIC. Consultor en el sector público y privado. 